\title{
Kurz- und langfristige Effekte der Ambulanten Medizinischen Rehabilitation für Patienten mit Asbestose
}

\section{Short- and Long-Term Effects of the Outpatient Medical Rehabilitation for Patients with Asbestosis}

Autoren

Institute
S. Dalichau' ${ }^{1}$ A. Demedts ${ }^{2}$, A. im Sande ${ }^{3}$, T. Möller ${ }^{1}$

Berufsgenossenschaftliche Unfall-Ambulanz und Reha-Zentrum am Airport Bremen

Facharztpraxis f. Lungen- und Bronchialheilkunde, Bremen

Berufsgenossenschaft Metall Nord Süd Bezirksverwaltung Bremen eingereicht 18.8.2009

akzeptiert 29.9.2009

\section{Bibliografie}

Dol http://dx.doi.org/ 10.1055/s-0029-1215245

Online-Publikation: 16. 11. 2009

Pneumologie 2010; 64:

163-170 @ Georg Thieme

Verlag KG Stuttgart · New York

ISSN 0934-8387

Korrespondenzadresse

Prof. Dr. rer. nat.

Stefan Dalichau

BG Unfall-Ambulanz u. Reha-

Zentrum am Airport Bremen

(Chefarzt: Dr. med.

Torsten Möller)

Industriestr. 3

28199 Bremen

sdalichau@ipl-bremen.de

\section{Zusammenfassung \\ $\nabla$}

Hintergrund: Die vorliegende Studie verfolgt die Fragestellung, ob und inwieweit Asbestosepatienten von einer intensiven ambulanten medizinischen Rehabilitation (AMR) profitieren können, die sich überwiegend aus bewegungs- und sporttherapeutischen Inhalten konstituiert und in Anlehnung an das Hamburger Modell insbesondere auf die Sicherung von Nachhaltigkeitseffekten ausgelegt ist.

Methode: Im Rahmen eines vorexperimentellen Studiendesigns durchliefen 104 männliche Asbestosepatienten im Lebensalter von $65,7 \pm 5$,5 Jahren 5-mal wöchentlich à $6 \mathrm{~h}$ über 3 Wochen Phase 1 (Aufbauphase) der AMR, bestehend aus standardisierten Inhalten der pneumologischen Rehabilitation. In der direkt folgenden Phase 2 (Stabilisierungsphase) der AMR absolvierten die Patienten einmal wöchentlich à $3 \mathrm{~h}$ über 12 Wochen weitere therapeutische Applikationen mit Schwerpunkt auf der Bewegungs- und Sporttherapie. Anschließend überführte die Reha-Einrichtung die Patienten in wohnortnahe Gesundheitssportgruppen (Erhaltungsphase). Die Effekte der AMR wurden zu Beginn (T1), am Ende der Aufbau(T2) und Stabilisierungsphase (T3) sowie 6 (T4) und 18 Monate (T5) nach T3 mittels eines geeigneten Assessments evaluiert.

Ergebnisse: Gemessen an T1 zeigten sich sowohl die körperliche Leistungsfähigkeit (6-min-Gehtest, Handkraft-Test, PWC-Test) als auch die gesundheitsbezogene Lebensqualität (SF-36) der Patienten in T2 signifikant verbessert. Während sich die Parameter „Vitalkapazität“ und „Ein-Sekunden-Kapazität“ über den Untersuchungszeitraum nicht veränderten, konnte der Messwert des arteriellen Sauerstoffdrucks in T2 ebenfalls statistisch bedeutend gesteigert werden. In T3 wurden die positiven Effekte bestätigt. 82 Patienten $(79 \%)$ des Kollektivs betrieben auch 6 und 18 Monate nach T3 noch regelmäßig Gesundheits-

\section{Abstract \\ $\nabla$}

Background: The aim of this study was to evaluate the effects of an outpatient medical rehabilitation (OMR) mainly composed of exercise therapy and sports for patients with asbestosis. Following the model of Hamburg the OMR focuses on keeping up lasting effects.

Methods: In the frame of a pre-experimental study 104 male patients aged 65,7 $\pm 5,5$ years suffering asbestosis carried out over a period of three weeks 5 times weekly $6 \mathrm{~h}$ at a time phase 1 of the OMR consisting of evidence-based contents of the pulmonary rehabilitation. Directly after that further therapeutic applications with the main focus on exercise therapy and sports were applied over a period of 12 weeks one time weekly $3 \mathrm{~h}$ at a time (phase 2). After phase 2 the rehabilitation centre led the patients into sports groups near at place of residence (phase 3 ). The effects of the OMR were evaluated at the beginning (T1), at the end of phase 1 (T2) and phase 2 (T3) as well as 6 (T4) and 18 months (T5) after T3 by means of a suitable assessment.

Results: Compared to T1 physical fitness (6-minute Walk Test, Hand-Force Test, PWC Test) as well as health-related quality of life (SF-36) of the patients were significantly improved in $\mathrm{T} 2$. Whereas the parameter "vital capacity" and "forced expiratory volume" showed no change over the period of investigation data of "oxygen partial pressure" was significantly increased, too. These positive effects could be confirmed in T3. 82 patients (79\%) were doing sports due to health regularly still 6 and 18 months after T3 and could preserve their health outcome in $\mathrm{T} 4$ and $\mathrm{T} 5$, while the effects of rehabilitation of the 22 patients breaking off any sporting activities wore off again to and even below starting condition in T1.

Conclusions: In spite of a restrictive pulmonary disease specific exercise therapy and sports are able to mobilize physical reserves of performance 
sport und konnten ihren Health Outcome in T4 und T5 erhalten, während die Reha-Effekte der 22 „Sportabbrecher“ wieder auf und sogar unter den Status quo ante in T1 zurückfielen.

Schlussfolgerungen: Trotz einer irreversiblen Lungenerkrankung mit restriktiver Funktionsstörung können gezielte bewegungs- und sporttherapeutische Interventionen eine Konditionierung im Sinne einer Aktivierung vorhandener körperlicher Leistungsreserven sowie konsekutiv eine Vergrößerung der Lebensqualität und Belastbarkeit im Alltag bewirken. Diese positiven Effekte lassen sich durch ein regelmäßiges Training (einmal wöchentlich) nachhaltig stabilisieren. Damit betonen die Ergebnisse die Notwendigkeit der Einbindung von Nachsorgestrategien in das Rehabilitationskonzept.

\section{Einleitung}

$\nabla$

Die Asbeststaublungenerkrankung ist eine Lungenfibrose und verursacht eine restriktive Lungenfunktionsstörung. Sie zeigt einen progredienten Verlauf mit irreversiblen morphologischen Veränderungen und mit möglicher Entwicklung eines Lungenoder Kehlkopfkrebs oder eines Mesothelioms des Rippenfells. Der Grad der „Schwere“ ist teils von der individuellen körpereigenen Reaktionsbereitschaft und teils von der aufgenommenen Asbeststaubmenge abhängig. Die Erkrankung wird frühestens etwa 10 Jahre nach Beginn der Asbestexposition klinisch und röntgenologisch manifest.

Von der Rehabilitation and Chronic Care Scientific Group der Europäischen Gemeinschaft für Lungenerkrankungen [1] werden für die notwendige pneumologische Rehabilitation folgende übergeordnete Ziele angegeben:

- Evaluierung u. Monitoring von Beeinträchtigungen als Folge einer Erkrankung der Atmungsorgane

- Linderung der (leistungs-)schwächenden physischen u. psychischen Symptome

- Wiederherstellung der bestmöglichen funktionellen Leistungsfähigkeit

- Förderung der sozialen Reintegration

Neben der traditionellen Durchführung der pneumologischen Rehabilitation in stationärer Form [2] ist bei definierten Krankheitsbildern wie z.B. der Lungenfibrose auch die wohnortnahe ambulante oder teilstationäre medizinische Rehabilitation bei Applikation identischer Therapieinhalte möglich [3]. Sie unterstützt eine engere Kooperation mit den weiterbehandelnden Ärzten und Betriebsmedizinern, die Einbindung Angehöriger und insbesondere die Optimierung einer Rehabilitationsnachsorge bzw. die Förderung der Nachhaltigkeit der Reha-Maßnahme [4]. Verschiedene Studien konnten die positiven kurzfristigen Effekte einer mehrwöchigen ambulanten pneumologischen Rehabilitation bei Patienten mit COPD mittels subjektiver und objektiver Messvariablen bereits belegen [5-7].

Im Auftrag verschiedener gesetzlicher Unfallversicherungsträger unter Federführung der Berufsgenossenschaft Metall Nord Süd Bezirksverwaltung Bremen wurde in Anlehnung an das Hamburger Modell [8] das Konzept für die Ambulante Medizinische Rehabilitation (AMR) bei Asbestose erstellt, durchgeführt und erste Ergebnisse evaluiert [9].

Nach einer Vorstellung der wesentlichen konzeptionellen Charakteristika der eingesetzten AMR bei Patienten mit Asbestose referiert der vorliegende Beitrag die kurz- und langfristigen Ergebnisse der begleitend durchgeführten Studie unter besonderer Berücksichtigung von Nachhaltigkeitseffekten, an denen sich der and induce an increasing quality of life as well as a higher resilience in activities of daily living. These positive effects could be stabilized persistently by a regular training one time weekly. That way the results emphasize the necessity to put strategies of aftercare into the concept of rehabilitation.

Erfolg jeder Rehabilitationsmaßnahme nach heutigem Verständnis bemessen lassen muss. Für eine umfassende Darstellung der für diese Rehabilitationsmaßnahme geltenden Rahmenbedingungen und gesetzlichen Voraussetzungen sei an dieser Stelle an die Ausführungen von Dalichau et al. [10] sowie Jeremie et al. [9] verwiesen.

\section{Konzept der AMR bei Asbestose \\ $\nabla$}

\section{Ziele}

Aufgrund der Irreversibilität der morphologischen Veränderungen und der resultierenden Funktionseinschränkungen lassen sich folgende Rehabilitationsziele definieren, deren Erfüllung langfristig der Erhöhung der Lebensqualität, der Fähigkeitsentwicklung zum Selbstmanagement und der Herausbildung der Eigenverantwortlichkeit bzgl. der Gesundheit bzw. der Erkrankung der Patienten dienen:

- Verbesserung der allgemeinen physischen und psychischen Leistungsfähigkeit

- Erhöhung der Belastbarkeit im Alltag

- Abnahme der Funktionseinschränkungen wegen Atemwegsbeschwerden bei gegebenen Aktivitäten des täglichen Lebens

- Steigerung des Selbstwertgefühls und des Selbstbewusstseins

- Förderung der sozialen Interaktion

- Krankheitsbewältigung, Krankheitsmanagement

- positive Lebenseinstellung (Beeinflussung des Kohärenzsinns im Sinne des Salutogenesemodells)

- Verbesserung des Kenntnisstands bzgl. der Erkrankung - gesundheitsbewusstere Lebensführung

Inhalte

Neben den Maßnahmen der Diagnostik (s.u.) konstituiert sich das Rehabilitationsprogramm aus folgenden Inhalten $[11,2,4]$ :

- Ärztliche Therapie

- Bewegungs-, Sport- und Trainingstherapie

- (Nordic-) Walking, Fahrrad-, Laufband- u. Oberkörperergometrie

- Medizinische Trainingstherapie - MTT [12]

- Kräftigungs- und Dehnungsgymnastik

- Wassergymnastik

- Entspannung, Gesprächsführung, Coping-Strategien [13]

- Techniken der Körpersensibilisierung, -wahrnehmung 


\begin{tabular}{|lllll} 
& Lebensalter (Jahre) & Körpergröße (cm) & Körpergewicht $\mathbf{( k g )}$ & Body-Mass-Index \\
\hline Mw \pm SD & $65,7 \pm 5,5$ & $175,4 \pm 6,4$ & $86,7 \pm 13$ & $27,9 \pm 3,8$ \\
\hline Min-Max & $53-78$ & $158-192$ & $52-114$ & $19,1-35,2$ \\
\hline
\end{tabular}

Tab. 1 Anthropometrische Daten der männlichen Untersuchungsgruppe $(n=104$; $\mathrm{Mw} \pm$ SD: Mittelwert u. Standardabweichung; Min-Max: Minimum-Maximum).

\begin{tabular}{|c|c|}
\hline Einschlusskriterien & Ausschlusskriterien \\
\hline $\begin{array}{l}\text { 1. BK-4103 mit MdE und Lungenfibrose, aber ohne } \\
\text { Symptome wie } \\
\text { - Atemnot oder häufige Infekte } \\
\text { - chronischer Husten } \\
\text { - reduzierte körperliche Belastbarkeit } \\
\text { - Sauerstoffuntersättigung des Blutes } \\
\text { 2. BK-4103 Fälle ohne MdE, aber mit Lungenfibrose oder } \\
\text { obstruktiver Ventilationsstörung } \\
\text { 3. statt eines stationären Heilverfahrens (bei Ablehnung } \\
\text { durch den Versicherten) }\end{array}$ & $\begin{array}{l}\text { Es wird keine AMR durchgeführt, wenn: } \\
\text { 1. gravierende Erkrankungen außerhalb der Lunge, } \\
\text { z. B. im Bereich des Herzens vorliegen und } \\
\text { 2. wenn diese nach Erkrankungen eindeutig einen } \\
\text { reduzierten Gesundheitszustand bestimmen bzw. } \\
\text { einen erheblichen therapeutischen oder pflegeri- } \\
\text { schen Aufwand erfordern oder } \\
\text { 3. wenn diese Erkrankungen die Teilnahme an den } \\
\text { therapeutischen Maßnahmen verhindern } \\
\text { (z.B. erhebliche degenerative Skeletterkrankungen) }\end{array}$ \\
\hline
\end{tabular}

\section{Ausschlusskriterien}

z. B. im Bereich des Herzens vorliegen und reduzierten Gesundheitszustand bestimmen bzw. einen erheblichen therapeutischen oder pflegeri(z.B. erhebliche degenerative Skeletterkrankungen)
Tab. 2 Ein- und Ausschlusskriterien zur Teilnahme an der Ambulanten Medizinischen Rehabilitation (AMR) bei Asbestose (BK: Berufskrankheit; MdE: Minderung der Erwerbsfähigkeit) (modifiziert n. [9]).
- Physiotherapie [14]

- in Gruppen- und Einzelbehandlung

- Atemgymnastik [15], Thoraxmobilisation, Manuelle Therapie

- Haltungsschulung

- Schulungen [16]

- zur Erkrankung/Gesundheit

- Ernährungsberatung

- Raucherentwöhnung

\section{Organisation und Durchführung}

Die AMR bei Patienten mit Asbestose beginnt mit einer dreiwöchigen Aufbauphase, die 13 zusammenhängende Therapietage (Montags-Freitags) umfasst, auf die sich die oben genannten Inhalte verteilen. Hinzu kommen jeweils ein Tag für die Eingangsund die Ausgangsuntersuchungen (=15 Tage). Samstage und Sonntage sind therapiefrei.

Die Aufbauphase findet grundsätzlich als Gruppenintervention statt, wobei bis zu 6 Patienten pro Gruppe aufgenommen werden. Die Patienten durchlaufen gemeinsam einen Rahmentherapieplan mit einer täglichen Nettotherapiezeit von 5 bis 6 Stunden, der inhaltlich sowie hinsichtlich von Umfang und Intensität der Einzelelemente auf die individuellen Bedürfnisse der Rehabilitanden abgestimmt wird. Die Gruppenorganisation steigert dabei die Compliance der Patienten, ermöglicht die Diskussion sowie den Erfahrungsaustausch und motiviert die Rehabilitanden zur Fortführung der AMR nach der Aufbauphase.

Eine Vielzahl von Studien belegt den Erfolg durchgeführter medizinischer Rehabilitationsleistungen, gleichzeitig wird aber auch dokumentiert, dass sich diese erzielten positiven Effekte im Laufe der Zeit wieder verringern $[17,18]$. Die für einen langfristigen Rehabilitationserfolg oft notwendigen Verhaltens- und Lebensstiländerungen sind Prozesse, die im Rahmen einer mehrwöchigen Rehabilitationsleistung eingeleitet, aber häufig nicht ausreichend gefestigt werden können. Aus diesen Gründen ist es wichtig und verpflichtend, Strategien zur Sicherung der Nachhaltigkeit von medizinischen Rehabilitationsleistungen anzuwenden, die auf der Grundlage des bio-psycho-sozialen Ansatzes der ICF die Eigenverantwortlichkeit des Rehabilitanden als selbstbestimmte Persönlichkeit betonen und als „Brücke“ zwischen zeit- lich begrenzter Rehabilitationsleistung und dem Alltagsleben des Rehabilitanden fungieren.

Nach Beendigung der dreiwöchigen Aufbauphase schließt sich infolge dessen die Stabilisierungsphase über einen Zeitraum von weiteren drei Monaten an, in denen die Patienten einmal wöchentlich jeweils 3 bis 4 Stunden Anwendungen der Medizinischen Trainingstherapie, des Ergometertrainings und der Wassergymnastik sowie der physiotherapeutischen Einzelbehandlung zugeführt werden. Zudem werden bei Bedarf zusätzliche Gesundheitsschulungen angeboten.

Zur langfristigen Sicherung und Erhaltung der Interventionseffekte auch nach Abschluss der 12-wöchigen Stabilisierungsphase werden die Patienten (auf freiwilliger Basis) von Mitarbeitern der Rehabilitationseinrichtung in wohnortnahe Gesundheits- bzw., wenn verfügbar, Lungensportgruppen überführt, um auf der Basis gezielter Bewegungsprogramme ein- bis zweimal wöchentlich à 60-90 min lebensbegleitend die Selbständigkeit hinsichtlich der Führung eines gesunden Lebensstils zu fördern (Erhaltungsphase) $[19,20]$.

Im Folgenden wird über kurz- und langfristige Effekte der vorgestellten AMR bei Patienten mit Asbestose berichtet.

\section{Methodik \\ $\nabla$}

\section{Patienten}

104 männliche Patienten mit Asbestose ( Tab. 1) nahmen nach ausführlicher Information freiwillig an der vorliegenden vorexperimentellen Untersuchung teil. Es galten die in Tab. 2 aufgeführten Ein- und Ausschlusskriterien zur Teilnahme an der AMR. 74\% der Patienten wiesen neben der Asbestose mindestens eine Komorbidität auf, 44,6\% zwei und 19,2\% der Reha-Teilnehmer mindestens drei Nebenerkrankungen. Medikamentös eingestellte sowie therapeutisch kontrollierte chronische Nebenerkrankungen der Patienten galten in diesem Zusammenhang a priori allerdings nicht als Ausschlusskriterien ( Tab. 3). Da die Inhalte sowie die Organisationsform dieser Rehabilitationsmaßnahme ungewohnt hohe körperliche Anforderungen an die Teilnehmer stellen, mussten alle Patienten vor Beginn der Intervention ihre „Rehafähigkeit“ nachweisen und sich einer medizinischen inter- 
Tab. 3 Häufigkeitsverteilung der Nebenerkrankungen der 104 Patienten der AMR bei Asbestose.

$\begin{array}{ll}\text { Nebenerkrankungen } & \text { Häufigkeit (in \%) } \\ \text { - Hypertonie } & 30,7 \\ \text { - COPD } & 27,8 \\ \text { - degenerativ bedingte Wirbelsäulen- } & 26 \\ \quad \text { beschwerden } & \\ \text { - degenerativ bedingte Beschwerden } & 16,3 \\ \quad \text { der Extremitätengelenke } & \\ \text { - koronare Herzkrankheit } & 14,4 \\ \text { - Diabetes Typ 2 } & 10,6 \\ \text { - Herz-Rhythmusstörungen } & 7,7 \\ \text { - sonstige } & 8,6\end{array}$

nistischen und lungenfachärztlichen Eignungsdiagnostik unterziehen.

\section{Messzeitpunkte und eingesetzte}

\section{Assessmentinstrumente}

Neben den routinemäßigen Verfahren der ärztlichen Diagnostik (insbesondere die mittels Bodyplethysmografie erhobenen Lungenfunktionsparameter: Vitalkapazität, Sekundenkapazität, Sauerstoffpartialdruck) und der physiotherapeutischen Befunderhebung wurden über einen Untersuchungszeitraum von 22 Monaten zu Beginn (T1) und nach Beendigung der dreiwöchigen Aufbauphase (T2) sowie nach Abschluss der Stabilisierungsphase (T3) nach weiteren 6 (T4) und nach weiteren 12 Monaten (T5) folgende als Verlaufskontrollen dienende standardisierte Assessmentinstrumente eingesetzt:

- Der 6-Minuten-Gehtest (6 MGT) zur Erfassung der Gehstrecke in Metern nach den Vorgaben der American Thoracic Society [21] als national und international anerkanntes Verfahren zur Beurteilung der körperlichen Leistungsfähigkeit insbesondere bei chronischen Lungenerkrankungen [11]. Gute bis sehr gute Ausprägungen von Reliabilität, Validität und Responsivität sind bereits in zahlreichen Studien nachgewiesen. Eine Übersicht hierzu findet sich in Büsching et al. [22].

- Der Physical Work Capacity Test (PWC) als etablierte Messmethode zur Quantifizierung der submaximalen Ausdauerleistungsfähigkeit auf dem Fahrradergometer [23] nach WHOSchema: Start bei 25 Watt; Steigerung der Wattzahl um 25 Watt alle 2 Minuten bei 60 Pedalumdrehungen/min bei drehzahlunabhängigen Ergometern; Dokumentation und Berechnung der erreichten Wattzahl bei einer aufgrund des hohen mittleren Lebensalters der Patienten festgelegten Herzfrequenz von 110/min durch Inter- oder Extrapolation.

- Messung der isometrischen Maximalkraft der Unterarm- und Handmuskulatur des dominanten Arms mittels Jamar-Dynamometrie: Der Patient befand sich in aufrecht sitzender Position. Der Oberarm der dominanten Seite war an den Oberkörper angelegt, der Ellbogen 90 Grad flektiert, der Unterarm bzgl. Pronation/Supination in Neutralstellung, das Handgelenk in 0-30 Grad Extension. Die Hand umgriff den an die individuellen anatomischen Voraussetzungen adaptierten Griff des Dynamometers. Auf Zeichen des Untersuchers drückte der Patient den unbeweglichen Griff zunehmend stärker bis maximal zusammen, ohne seine Körperposition zu verändern, und hielt die Spannung für ca. 2 -3 Sekunden. Anschließend wurde die Muskelkontraktion wieder gelöst und der auf der Skala des Geräts angezeigte Messwert (in kg) vom Untersucher notiert. Dieser Vorgang wurde insgesamt dreimal durchgeführt. Der Mittelwert der drei Messungen ging in die Auswertung ein. Die in mehreren Studien ermittelten Korrelationskoeffizienten für die Intra- und Interrater Reliabilität sowie die Validität mit $r=0,82-0,99$ qualifizieren die JamarDynamometrie zur Erfassung der Handkraft als Assessmentinstrument für den wissenschaftlichen Einsatz [24,25]. Zudem gelten die erfassten Messwerte als aussagekräftig für die Bewältigung von Alltagsaktivitäten insbesondere bei chronischen Lungenerkrankungen [26].

- Der SF-36 zur Erfassung der gesundheitsbezogenen Lebensqualität: Der SF-36 zur Dokumentation eines mehrdimensionalen Gesundheitsprofils stellt ein Verfahren dar, dessen psychometrische Qualität national und international ausreichend unter Beweis gestellt wurde [27] und welches sich auch im Rahmen von vergleichenden und Evaluationsstudien sehr gut einsetzen lässt [28]. Es werden hierbei 8 Dimensionen der psychischen, körperlichen und sozialen Ebene infolge einer schriftlichen Befragung (36 Fragen) des Patienten erfasst und jeweils als Prozentangaben von 0 - maximal 100\% Lebensqualität quantifiziert.

Die Darstellung weiterer in dieser Untersuchung eingesetzter Assessmentinstrumente an dieser Stelle würde den Rahmen dieser Publikation übersteigen. Die Ergebnisse werden separat und in Kürze veröffentlicht.

\section{Statistik \\ $\nabla$}

Die Auswertung der erfassten Daten erfolgte mit dem Computerprogramm SPSS Base 13.0 für Windows. Zu Beginn der Auswertung wurde der Datensatz durch eine Plausibilitätskontrolle mit dem System überprüft und die Normalverteilung der Stichprobe im Hinblick auf die jeweiligen zu untersuchenden abhängigen Variablen mittels des Kolmogorov-Smirnov-Tests bestätigt.

Neben den Verfahren der Deskriptiven Statistik wurde die einfaktorielle Varianzanalyse mit Messwiederholung und posthoc-Testdesign bei vier bzw. fünf Messzeitpunkten eingesetzt sowie die Korrelationsanalysen nach Pearson und nach Spearman-Rho durchgeführt.

\section{Ergebnisse}

Die Ergebnisse der motorischen Tests ( $\bullet$ Tab. 4) signalisieren in allen untersuchten Messvariablen signifikante Veränderungen der Gesamtstichprobe von T1 zu T2, die am Ende der Stabilisierungsphase (T3) tendenziell weiter ausgebaut und sowohl nach weiteren 6 Monaten als auch im Follow up nach weiteren 12 Monaten (T5) erhalten werden können. In Orientierung an den Normwerten können die Messwerte als Verbesserungen interpretiert werden, die mit Effektstärken zwischen 0,4 und 0,72 als moderat bis stark einzustufen sind [22]. Während sich die direkten Lungenfunktionsparameter Vitalkapazität und die Ein-Sekundenkapazität unverändert zeigen, sind ebenfalls statistisch bedeutsame Steigerungen des arteriellen Sauerstoffpartialdrucks sowohl von T1 zu T2 als auch zu T3 festzustellen, die in T5 nur leicht abfallen. Analog zu den positiven Resultaten der motorischen Tests zeigt sich die Entwicklung der subjektiv wahrgenommenen gesundheitsbezogenen Lebensqualität ( $\boldsymbol{Q}$ Abb. 1). Alle 8 Subskalen des SF-36 demonstrieren ausgehend von T1 eine signifikante Zunahme in T2, die in T3, T4 und T5 stabilisiert werden kann und deren durchschnittliche absolute Ausprägung in den 
Tab. 4 Ergebnisse des 6-Minuten-Gehtests (6 MGT), des PWC-Tests 110, der isometrischen maximalen Handkraft und der Lungenfunktionsparameter Inspiratorische Vitalkapazität (VK IN), Ein-Sekunden-Kapazität (FEV1) und Sauerstoffpartialdruck (pO2) in Ruhe als Mittelwerte \pm Standardabweichung und Effektstärken (ES) sowie im Vergleich zu in der Literatur beschriebenen Normwerten; ${ }^{*} p<0,05$ kennzeichnet statistisch signifikante Unterschiede der jeweiligen Testzeitpunkte (T2-T5) zu T1.

\begin{tabular}{|c|c|c|c|c|c|c|c|}
\hline$N=104$ & & T1 & T2 & T3 & T4 & T5 & Normwerte \\
\hline \multirow[t]{2}{*}{$6 \mathrm{MGT}$} & $\mathrm{m}$ & $455,7 \pm 72,8$ & $499,7^{*} \pm 64,7$ & $505,5^{*} \pm 68,6$ & $497,2^{*} \pm 71,6$ & $491,7^{*} \pm 73,2$ & $377-530[29]$ \\
\hline & ES & - & 0,68 & 0,72 & 0,57 & 0,49 & - \\
\hline \multirow[t]{3}{*}{ PWC 110} & Watt & $85,8 \pm 30,5$ & $101,3^{*} \pm 31,7$ & $101,5^{*} \pm 29,4$ & $100,1^{*} \pm 29,8$ & $98,6^{*} \pm 30,5$ & \multirow{2}{*}{$1,5-1,99$ PWC 130 [30] } \\
\hline & Watt $/ \mathrm{kg}$ & $0,99 \pm 0,34$ & $1,18^{*} \pm 0,36$ & $1,19^{*} \pm 0,34$ & $1,17^{*} \pm 0,35$ & $1,15^{*} \pm 0,36$ & \\
\hline & ES & - & 0,51 & 0,51 & 0,46 & 0,42 & - \\
\hline \multirow[t]{2}{*}{ Handkraft } & $\mathrm{kg}$ & $46,2 \pm 9,2$ & $48,9^{*} \pm 9,4$ & $49,8^{*} \pm 10,2$ & $49,5^{*} \pm 10,8$ & $49,1^{*} \pm 10,1$ & $41,3 \pm 9,3[31]$ \\
\hline & ES & - & 0,4 & 0,5 & 0,46 & 0,42 & - \\
\hline \multirow[t]{2}{*}{ VK IN } & I & $3,07 \pm 0,72$ & $3,06 \pm 0,77$ & $3,06 \pm 0,75$ & \multirow{6}{*}{ in T4 nicht erhoben } & $3,04 \pm 0,75$ & $4,14 \pm 0,92[32]$ \\
\hline & ES & - & $-0,01$ & $-0,01$ & & $-0,04$ & - \\
\hline \multirow[t]{2}{*}{ FEV1 } & I & $2,36 \pm 0,68$ & $2,33 \pm 0,72$ & $2,35 \pm 0,68$ & & $2,35 \pm 0,71$ & $3,09 \pm 0,84[32]$ \\
\hline & ES & - & $-0,04$ & $-0,01$ & & $-0,01$ & - \\
\hline \multirow{2}{*}{$\begin{array}{l}\text { pO2 } \\
\text { (in Ruhe) }\end{array}$} & $\mathrm{mmHg}$ & $67,3 \pm 9,9$ & $72,1^{*} \pm 11,7$ & $73,9^{*} \pm 11,4$ & & $71,1^{*} \pm 12,4$ & $71[32]$ \\
\hline & ES & - & 0,48 & 0,66 & & 0,38 & - \\
\hline
\end{tabular}

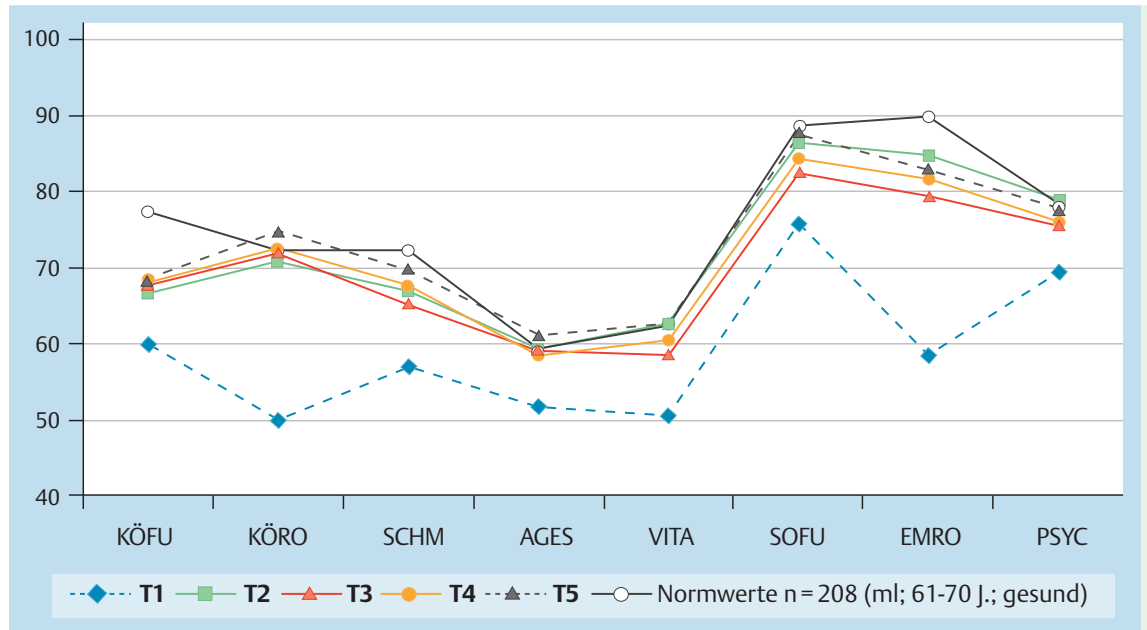

Abb. 1 Entwicklung der gesundheitsbezogenen Lebensqualität als Mittelwerte (in \%) unter Berücksichtigung der acht SF-36 Skalen vom 1. bis zum 5. Messzeitpunkt ( $T 1 \rightarrow T 5 ; n=104)$ im Vergleich zu einer gesunden alters- und geschlechtsgleichen Referenzgruppe [27]; KÖFU = Körperl. Funktionsfähigkeit; KÖRO = Körperl. Rollenfunktion; SCHM = Körperl. Schmerzen; AGES = Allgemeine Gesundheit; VITA = Vitalität; SOFU = Soziale Funktionsfähigkeit; EMRO $=$ Emotionale Rollenfunktion; PSYC $=$ Psychisches Wohlbefinden.

Skalen der Körperlichen Rollenfunktion, der Allgemeinen Gesundheit, der Vitalität und des Psychischen Wohlbefindens sogar annährend die Ergebnisse der Normwerte der gesunden Referenzgruppe erreicht.

Von 104 Patienten der Gesamtstichprobe konnten 82 Probanden (79\%) nach Ende der Stabilisierungsphase zu einer regelmäßigen Fortführung der sportlichen Aktivität ( $\geq$ einmal wöchentlich) in Wohnortnähe motiviert werden, die über 18 Monate im Durchschnitt 2,3-mal/Woche ausgeübt wurde. Eine Übersicht der durchgeführten Bewegungsangebote gibt $\bullet$ Abb. 2. Hingegen beendeten 22 Patienten (21\%) nach der Stabilisierungsphase (T3) jegliche zielgerichtete Sportausübung aus unterschiedlichen Gründen: keine Motivation/Zeit $(n=11)$, Krankheit/Krankheit der Lebenspartnerin $(\mathrm{n}=10)$, keine geeignete Bewegungsform in Wohnortnähe $(\mathrm{n}=1)$.

Wird nach Sportausübung nach der Stabilisierungsphase (ja/ nein) differenziert, ist zu erkennen, dass die Sportgruppe die positiven Steigerungsraten in T3 im 4. und 5. Testzeitpunkt nicht nur konsolidieren, sondern tendenziell sogar noch weiter steigern kann. Das gilt sowohl für die motorischen Tests und den Sauerstoffpartialdruck ( Tab.5) als auch für die Erfassung der gesundheitsbezogenen Lebensqualität ( $\bullet$ Abb.3). Im Gegensatz dazu remittieren die Messwerte in der „Kein-Sport-Gruppe“ bedeutend und nähern sich in T4 wieder deutlich dem Ausgangsni-

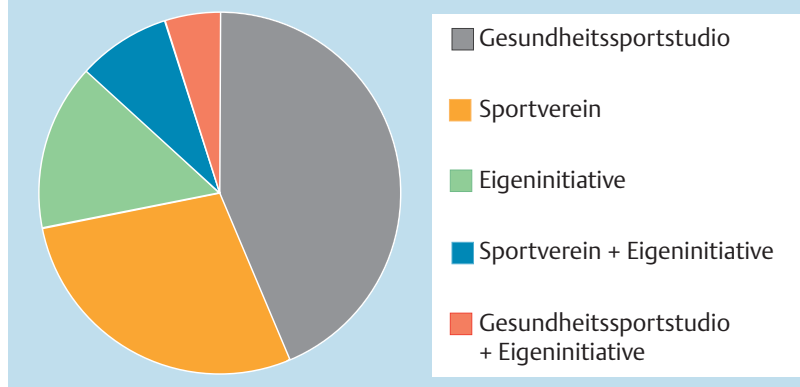

Abb.2 Häufigkeitsverteilung (in \%) hinsichtlich der sportlichen Aktivität in der Erhaltungsphase zum 5. Messzeitpunkt (T5) bei 82 Patienten der AMR bei Asbestose.

veau in T1 und fallen 12 weitere Monate später (T5) sogar unter den Status quo ante zurück.

Die Ergebnisse der Korrelationsanalysen ( Tab.6) demonstrieren einen geringen bis mäßigen, statistisch bedeutenden, leistungsmindernden Einfluss eines höheren Lebensalters auf die Testergebnisse des 6-min-Gehtests und der Handkraftmessung sowie eines erhöhten Body-Mass-Index (BMI) auf die Ausdauerleistungsfähigkeit beim PWC-Test. Hingegen besitzen die Art und insbesondere die Anzahl der Nebenerkrankungen der Pa- 
Tab. 5 Ergebnisse des 6-Minuten-Gehtests (6 MGT), des PWC-Tests 110 und der isometrischen maximalen Handkraft als Mittelwerte \pm Standardabweichung differenziert nach Patienten, die 6 Monate nach T3 mindestens einmal wöchentlich eine sportliche Aktivität von $\geq 1$ h absolvierten (Sportgruppe; $\mathrm{n}=82$ ), und Patienten, die in dieser Zeit keinen Sport ausübten (kein Sport; $n=22$ ); ${ }^{*}<<0,05$ kennzeichnet statistisch signifikante Unterschiede der jeweiligen Testzeitpunkte (T2-T5) zu T1

\begin{tabular}{lllllll} 
& & T1 & T2 & T3 & T4 & T5 \\
6 MGT & Sportgruppe in T4 u. T5 & $456,7 \pm 69,7$ & $500,1^{*} \pm 75,8$ & $508,3^{*} \pm 68,6$ & $509,8^{*} \pm 70,2$ & $506,8^{*} \pm 72,3$ \\
(in m) & kein Sport in T4 u. T5 & $453,8 \pm 78,5$ & $497,8^{*} \pm 87,4$ & $499,3^{*} \pm 78,6$ & $464,5 \pm 89,2$ & $444,8 \pm 87,5$ \\
\hline PWC 110 & Sportgruppe in T4 u. T5 & $86,4 \pm 31,1$ & $101,8^{*} \pm 31,2$ & $103,2^{*} \pm 28,4$ & $105,1^{*} \pm 27,8$ & $105,7^{*} \pm 28,1$ \\
(in Watt) & kein Sport in T4 u. T5 & $85,8 \pm 29,4$ & $101,7^{*} \pm 32,8$ & $99,4^{*} \pm 30,8$ & $88,1 \pm 32,4$ & $76,9^{*} \pm 27,1$ \\
\hline Handkraft & Sportgruppe in T4 u. T5 & $45,5 \pm 9,5$ & $48,6^{*} \pm 9,1$ & $49,6^{*} \pm 10,3$ & $50,6^{*} \pm 10,5$ & $50^{*} \pm 9,5$ \\
(in kg) & kein Sport in T4 u. T5 & $47,8 \pm 10,8$ & $50,1^{*} \pm 8,1$ & $50,8 \pm 10,8$ & $47 \pm 11,2$ & $44,7^{*} \pm 10,8$ \\
\hline PO2 (in Ruhe) & Sportgruppe in T4 u. T5 & $67,4 \pm 10,6$ & $72,8^{*} \pm 11,7$ & $73,3^{*} \pm 10,1$ & in T4 nicht erhoben & $73,4^{*} \pm 10,3$ \\
\hline (mmHg) & kein Sport in T4 u. T5 & $66,8 \pm 8,3$ & $71,1^{*} \pm 11,3$ & $75^{*} \pm 11,8$ & & $66,7 \pm 9,7$ \\
\hline
\end{tabular}

Tab. 6 Zusammenhänge zwischen den untersuchten Parametern und ausgewählten Faktoren dargestellt als Korrelationskoeffizienten $(r) ;{ }^{*} \mathrm{p}<0,05$.

\begin{tabular}{|c|c|c|c|c|c|}
\hline $\mathbf{r}$ & $\begin{array}{l}\text { Lebensalter } \\
\text { ( } \mathrm{T} 1 \text { bis T5) } \\
\mathrm{n}=104\end{array}$ & $\begin{array}{l}\text { Body-Mass-Index } \\
\text { (T1 bis T5) } \\
n=104\end{array}$ & $\begin{array}{l}\text { Anzahl der Neben- } \\
\text { erkrankungen } \\
\text { (T1 bis T5) } n=104\end{array}$ & $\begin{array}{l}\text { Anzahl wöchent- } \\
\text { liches Sporttreiben } \\
\text { (T4) } n=82\end{array}$ & $\begin{array}{l}\text { Anzahl wöchent- } \\
\text { liches Sporttreiben } \\
\text { (T5) } n=82\end{array}$ \\
\hline 6-min-Gehtest & $-0,28$ bis $-0,43^{*}$ & $-0,09$ bis $-0,19$ & $-0,08$ bis $-0,13$ & $0,23^{*}$ & 0,14 \\
\hline Handkraft & $-0,26$ bis $-0,39^{*}$ & 0,11 bis 0,19 & $-0,04$ bis 0,09 & 0,09 & 0,06 \\
\hline PWC (Watt/kg) & $-0,06$ bis $-0,18$ & $-0,22$ bis $-0,27^{*}$ & $-0,03$ bis 0,11 & 0,21 & 0,15 \\
\hline SF-36 & $-0,04$ bis $-0,12$ & $-0,07$ bis $-0,17$ & $-0,05$ bis 0,03 & 0,14 & 0,08 \\
\hline
\end{tabular}

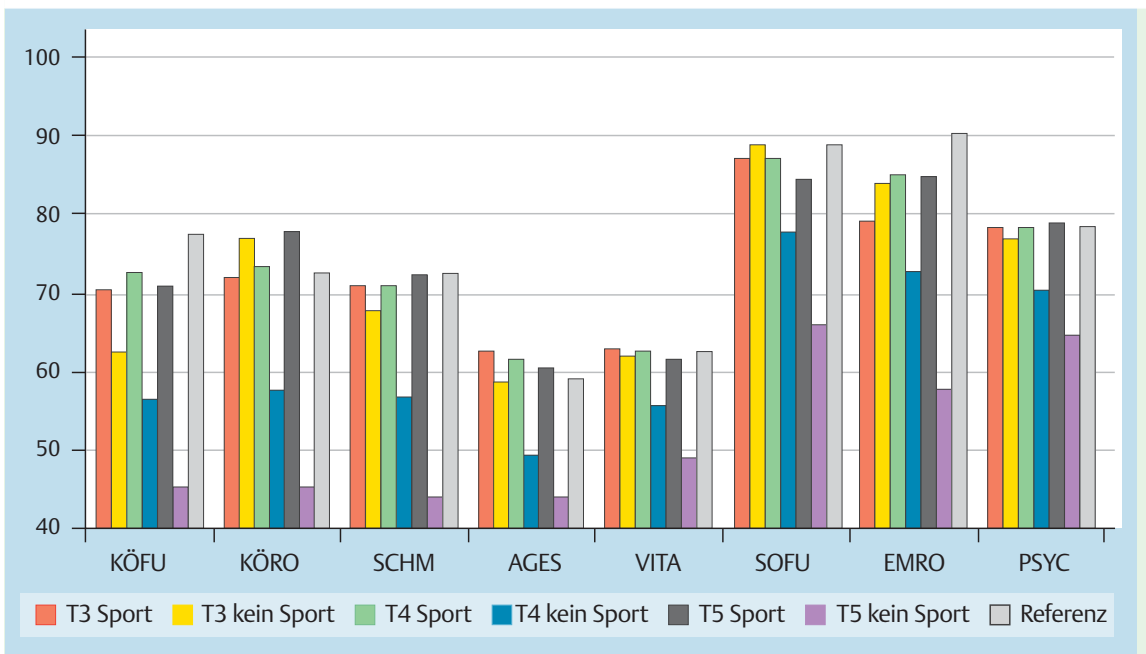

Abb. 3 Entwicklung der gesundheitsbezogenen Lebensqualität als Mittelwerte (in \%) unter Berücksichtigung der acht SF-36 Skalen vom 3. bis zum 5. Messzeitpunkt (T3 $\rightarrow$ T5) differenziert nach Patienten, die 6 und 18 Monate nach T3 mindestens einmal wöchentlich eine sportliche Aktivität von $\geq 1 \mathrm{~h}$ absolvierten (Sport; $\mathrm{n}=82$ ), und Patienten, die in dieser Zeit keinen Sport ausübten (kein Sport; $\mathrm{n}=22$ ) im Vergleich zu einer gesunden alters- und geschlechtsgleichen Referenzgruppe ( $n=208$; aus [27]); KÖFU = Körperl. Funktionsfähigkeit; KÖRO = Körperl. Rollenfunktion; SCHM = Körperl. Schmerzen; AGES = Allgemeine Gesundheit; VITA = Vitalität; SOFU = Soziale Funktionsfähigkeit; $\mathrm{EMRO}=$ Emotionale Rollenfunktion; PSYC = Psychisches Wohlbefinden.

tienten keine nachweisbare Relevanz hinsichtlich der Ausprägung der Messwerte. In der Sportgruppe führt eine Zunahme an wöchentlichen Übungsterminen zu einer geringen statistisch signifikanten Steigerung im 6 MGT in T4. Die Ausdauerleistungsfähigkeit und die gesundheitsbezogene Lebensqualität werden durch eine Frequenzsteigerung tendenziell positiv beeinflusst.

\section{Diskussion}

Obwohl es sich bei der vorgestellten Evaluation um eine vorexperimentelle Untersuchung, also eine nicht kontrollierte Studie handelt und somit die positiven Ergebnisse nicht eindeutig als durch die Rehabilitationsmaßnahme induziert statistisch abgesichert werden können, liegt die Vermutung nahe, dass Asbestose Patienten mit den geltenden Ein- und Ausschlusskriterien ( Tab. 2) von dieser Form der Gesundheitsförderung kurz- und auch langfristig Benefit erfahren. Trotz einer strukturell irreversi- blen restriktiven Lungenerkrankung können gezielte bewegungs- und sporttherapeutische Interventionen eine Konditionierung im Sinne einer Mobilisierung vorhandener körperlicher Leistungsreserven sowie konsekutiv eine Vergrößerung der Lebensqualität und Belastbarkeit im Alltag bewirken. Zwar zeigen die klassischen Lungenfunktionsparameter „Vitalkapazität“ und „Ein-Sekunden-Kapazität“ keine Veränderungen über den Untersuchungszeitraum, der Sauerstoffpartialdruck als Funktionskennwert der Sauerstoffaufnahme hingegen scheint von der Therapiemaßnahme zu profitieren. Analog zu der nachweisbaren verbesserten Ausdauer- und körperlichen Leistungsfähigkeit ist auch die Erhöhung des Sauerstoffpartialdrucks im Sinne einer signifikanten Funktionsverbesserung als Adaptation an die Anforderungen der AMR zu interpretieren [19,32]. Die Effektstärke der AMR wird dabei eher vom Lebensalter und dem BMI der Patienten und weniger von der Art und der Anzahl der Komorbiditäten beeinflusst, sofern die Nebenerkrankungen medikamentös adäquat eingestellt und somit beherrschbar sind. 
Die AMR bei Asbestose dient als Erweiterung und Ergänzung der stationären Heilbehandlung und sieht die langfristige therapeutische Begleitung der Patienten als fokussiertes Rehabilitationsziel. Studien, die die Bedingungen einer längerfristigen Bindung an regelmäßige sportliche Aktivität analysierten [33-37], zeigen eindeutig, dass die Drop-out Raten 6 Monate nach Aufnahme eines Gesundheitsförderungsprogramms bei ca. 50\% liegen. Göhl [38] macht zudem auf die bisher unzureichend funktionierende Rehabilitationskette in der Therapie von Patienten mit chronischen Lungenerkrankungen aufmerksam.

In der dreiwöchigen Aufbau- und dreimonatigen Stabilisierungsphase wird beabsichtigt, die Teilnehmer zur Aufnahme einer gesundheitssportlichen Aktivität und zu einer gesünderen Lebensform zu motivieren, die lebensbegleitend und praktikabel in den Alltag zu integrieren sein muss. Wichtig ist dabei, dass die sportliche Gemeinschaft in der Gruppe (Familie, Verein) unter Personen ähnlicher Leistungsstärke (Förderung der Gruppendynamik) stattfindet. Zudem darf die körperliche Beanspruchung weder zu intensiv noch zu häufig durchgeführt werden, um den in der Regel nicht an regelmäßige sportliche Aktivität gewohnten Teilnehmer nicht zu überfordern und die Freude an der Bewegung nicht in einen Bewegungszwang ausarten zu lassen. Bewährt hat sich für das erste Jahr ein regelmäßiges Training von einmal wöchentlich ca. 60-90 min [39], was sich infolge eines wachsenden Gesundheitsempfindens des Teilnehmers durch Eigeninitiative ausweiten kann.

Die Ergebnisse nach 6 sowie weiteren 12 Monaten wohnortnahen Sporttreibens sprechen für einen Konsolidierungseffekt der positiven Rehaergebnisse bei einer durchschnittlichen Frequenz von zweimal wöchentlich, wobei die Resultate der prüfenden Statistik belegen, dass bereits ein einmal in der Woche durchgeführtes Training in der Erhaltungsphase die Rehaeffekte sichert. Weitere Zuwächse durch eine Zunahme der wöchentlichen Häufigkeit sind nur für die Ausdauerleistungsfähigkeit in T4 geringfügig signifikant. Wird die sportliche Aktivität dagegen nach der Stabilisierungsphase nicht fortgeführt, remittieren die positiven Rehaeffekte bereits sechs Monate später und fallen nach weiteren 12 Monaten sogar unter den Status quo ante in T1 zurück. Somit bestätigen die vorliegenden Ergebnisse der „Sportabbrecher“ die beobachteten Phänomene der eingeschränkten Nachhaltigkeit von Rehabilitationsmaßnahmen $[17,18,40]$ und unterstreichen die Notwendigkeit der Einbindung von Strategien zur Sicherung der langfristigen Fortsetzung körperlicher Aktivität sowie eines gesunden Lebensstils in das Rehabilitationskonzept.

Doch wie können Rehabilitanden ermuntert und befähigt werden, selbst Strategien und Kompetenzen zur Überwindung ihrer beeinträchtigten Aktivitäten/Teilhabe zu entwickeln und - auch gemeinsam mit anderen - erfolgreich umzusetzen? Geeignete Ansätze gruppieren sich um die Begriffe „Fähigkeiten und Ressourcen“. Unter Kompetenzen werden Fähigkeiten verstanden, die benötigt werden, um den individuellen Anforderungen gerecht zu werden. Auf der persönlichen Ebene geht es zunächst darum, die eigene Situation zu erkennen und zu akzeptieren und den Wunsch und die Bereitschaft, diese Situation selbst in die Hand zu nehmen und aktiv zu gestalten. Hierzu gilt es, den Fokus auf vorhandene Ressourcen und Kompetenzen zu richten, um so das Selbstwertgefühl hervorzuheben, das Kontrollbewusstsein zu stärken und die Bewältigungsstrategien zu erlernen.

Auf der Ebene der Teilhabe gilt es, die Rehabilitanden für die Erkenntnis zu sensibilisieren, dass auch andere Personen als die Rehabilitanden ähnlich gelagerte Probleme haben. Aus der Interaktion mit diesen können gemeinsame Interessen entwickelt und Ziele formuliert werden. Individueller Veränderungswillen und gemeinsam formulierte Ziele gehen in die Strategie zur Zielerreichung ein. Hierzu gehört, ebenso Einfluss auf Kontextfaktoren zu nehmen wie auch Netzwerke und Unterstützungsstrukturen zu nutzen, um die eigenen Interessen umzusetzen.

In diesem Zusammenhang ist insbesondere die Motivationsförderung während des Rehabilitationsprozesses von entscheidender Bedeutung und ein unverzichtbarer Bestandteil der Rehabilitationsleistungen. Durch das Aufzeigen von Wegen der Eigeninitiative und die Ermunterung des Rehabilitanden, geeignete nachsorgende Aktivitäten in seiner Umgebung wahrzunehmen, wird die Rückkehr des Rehabilitanden in seinen Lebensalltag vorbereitet.

In Anlehnung an den Praxisleitfaden „Strategien zur Sicherung der Nachhaltigkeit von Leistungen zur medizinischen Rehabilitation" der Bundesarbeitsgemeinschaft für Rehabilitation [41] werden im Rahmen der AMR bei Asbestose zur Erreichung langfristiger Effekte zahlreiche Maßnahmen in der therapeutischen Praxis routinemäßig eingesetzt ( $\bullet$ Tab. 7). Die fortlaufende Dokumentation und Evaluation der AMR bei Asbestose erlaubt dabei die notwendige stetige Weiterentwicklung und Optimierung des derzeitigen Konzepts.

Tab. 7 Maßnahmen zur Sicherung der Nachhaltigkeit von Rehabilitationseffekten unter Berücksichtigung der drei Phasen der AMR bei Asbestose.

\begin{tabular}{|c|c|c|}
\hline vor u. während der Aufbauphase & in der Stabilisierungsphase & in der Erhaltungsphase \\
\hline $\begin{array}{l}\text { Info-Veranstaltung: } \\
\text { - erste Kontaktaufnahme } \\
\text { - erste Sensibilisierung } \\
\text { - Information } \\
\text { Medizinische Untersuchung: } \\
\text { - Prüfung auf Rehafähigkeit } \\
\text { - Stellung der Rehaprognose } \\
\text { - zweite Sensibilisierung } \\
\text { T1, Aufbauphase, T2: } \\
\text { - Realisierung der Rehaeffekte } \\
\text { - Einordnung physiologischer u. psycholo- } \\
\text { gischer Reaktionen der Patienten durch } \\
\text { Einzel- u. Gruppengespräche } \\
\text { - Motivation u. Einsicht durch Verbesserung der } \\
\text { Lebensqualität } \\
\text { - Erhöhung der Selbstwirksamkeitserwartung }\end{array}$ & $\begin{array}{l}\text { - Stabilisierung und Rhythmusbildung durch } \\
\text { mittelfristige regelmäßige Exposition in Form } \\
\text { einmal wöchentlichen körperlichen Trainings } \\
\text { - Prinzip der individuellen Beratung } \\
\text { - Benennung eines Ansprechpartners in der } \\
\text { Reha-Einrichtung für die Patienten } \\
\text { - Analyse der bevorzugten Form der körper- } \\
\text { lichen Aktivität des Patienten durch Einzel- } \\
\text { gespräche } \\
\text { - Auswahl u. Kontaktaufnahme zur ausgewähl- } \\
\text { ten wohnortnahen Sportstätte durch die Reha- } \\
\text { Einrichtung } \\
\text { - Prüfung der Qualität der Sportstätte durch } \\
\text { die Reha-Einrichtung } \\
\text { - Motivationsförderung durch T3-Messergeb- } \\
\text { nisse (Bestätigung durch Objektivierung) }\end{array}$ & $\begin{array}{l}\text { - Einrichtung einer Hotline } \\
\text { - Versendung eines Newsletters der Reha- } \\
\text { Einrichtung } \\
\text { - Empfehlung von themenbezogenen Internet- } \\
\text { adressen und Informationsquellen } \\
\text { - Überprüfung der Qualität der wohnortnahen } \\
\text { Sportstätte durch Messung in T4 u. T5, ggf. } \\
\text { Nachbesserung durch Kontaktaufnahme } \\
\text { - Sensibilisierung der „Sportabbrecher“ durch } \\
\text { Einzelgespräche } \\
\text { - Motivationsförderung durch T4- u. T5-Mess- } \\
\text { ergebnisse bei Patienten, die Sport treiben }\end{array}$ \\
\hline
\end{tabular}


Die bisherigen Ergebnisse lassen sich als vielversprechend charakterisieren und demonstrieren die Bedeutung einer „Therapiestraße" mit der Betonung auf Maßnahmen zur Sicherung von Nachhaltigkeit und somit zur Erlangung von mehr Gesundheit und mehr Lebensqualität für Menschen mit einer chronischen Erkrankung.

\section{Interessenkonflikte \\ $\nabla$}

Keine angegeben.

\section{Literatur}

1 European Respiratory Society, Rehabilitation and Chronic Care group. Pulmonary Rehabilitation in Chronic Obstructive Pulmonary Disease (COPD) with recommendations for its use. Eur Respir J 1992; 5: 266 275

2 Bergmann K-C, Fischer J, Schmitz $M$ et al. Die stationäre pneumologische Rehabilitation für Erwachsene: Zielsetzung - diagnostische und therapeutische Standards - Forschungsbedarf. Pneumologie 1997; 51: $523-532$

3 Dalichau S, Demedts A, Jeremie U, Möller T. Die intensivierte ambulante Rehabilitation für Arbeitnehmer der Metallindustrie mit Asbestose Erste Ergebnisse der Pilotphase. In: VDR, Hrsg. 13. Rehabilitationswissenschaftliches Kolloquium. Selbstkompetenz - Weg und Ziel der Rehabilitation vom 8. bis 10. März in Düsseldorf, Band 52. Frankfurt a. M: VDR, 2004: 499-500

4 Bundesarbeitsgemeinschaft für Rehabilitation, Hrsg. Rahmenempfehlungen zur ambulanten pneumologischen Rehabilitation. Frankfurt a. M: BAR, 2009

5 du Moulin M, Taube K, Wegscheider Ket al. Home-based exercise training as maintenance after outpatient pulmonary rehabilitation. Respiration 2009; 77: 139-145

6 Ong KC, Wong WP, Jailani AR et al. Effects of a pulmonary rehabilitation programme on physiologic and psychosocial outcomes in patients with chronic respiratory disorders. Ann Acad Med Singapore 2001; 30: $15-21$

7 Taube K, Behnke M, Magnusson H. Effekte einer ambulanten/teilstationären Rehabilitation bei Patienten mit COPD. Pneumologie 2003; 57: $1-102$

8 Taube K. Ambulante/teilstationäre pneumologische Rehabilitation Kurzfassung des Konzeptes. Hamburg: Atem-Reha GmbH, 2003

9 Jeremie U, Grützmacher J, Taube K et al. Verbesserung der Rehabilitation von Asbestosen durch die Einführung eines ambulanten Rehabilitationsprogramms - Erste Ergebnisse. Arbeitsmed Sozialmed Umweltmed 2006; 41: 416-423

10 Dalichau S, Ameling H, Münzner L, Oltsch C. Ambulante Rehabilitation bei restriktiven Atemwegserkrankungen unter Berücksichtigung der Asbestose. In: Dalichau S, Scheele K, Hrsg. Prävention und Rehabilitation durch Sport. Butzbach/Griedel: Afra, 2006: 115-134

11 American Thoracic Society and European Respiratory Society. Statement on Pulmonary Rehabilitation. Am J Resp Crit Care Med 2006; 173 : $1390-1413$

12 Müller C. Trainingstherapie in der pneumologischen Rehabilitation was ist gesichert - wo besteht Forschungsbedarf? Atemw-Lungenkrkh 2004; 30: $422-425$

13 Schwiersch M. Psychologischer Support in der pneumologischen Rehabilitation. Atemw- Lungenkrkh 2004; 30: 450-455

14 Steier J. Physiotherapie bei Atemwegs- und Lungenerkrankungen. Atemw-Lungenkrkh 2004; 30: 426-431

15 Rühle $K H$, Nilius G. Training der Atemmuskulatur bei Patienten mit chronisch obstruktiver Lungenerkrankung. Atemw-Lungenerkrkh $2005 ; 31: 533-538$
16 Dhein Y. Patientenschulung - was ist evidenzbasiert? Atemw-Lungenkrkh 2004; 30: 446-449

17 Deck R, Raspe H. Nachsorgeempfehlungen und ihre Umsetzung im Anschluss an Rehabilitation. In: Deck R, Glaser-Möller N, Mittag O, Hrsg. Rehabilitation und Nachsorge. Bedarf und Umsetzung. Lage: Jacobs, 2004: $55-70$

18 Haaf H-G. Ergebnisse zur Wirksamkeit der Rehabilitation. Rehabilitation $2005 ; 44: 259-276$

19 Rost R. Sport- und Bewegungstherapie bei Inneren Krankheiten. 3. Aufl. Köln: Dt. Ärzte-Verlag, 2005

20 Worth H, Meyer A, Folgering $H$ et al. Empfehlungen der Deutschen Atemwegsliga zum Sport und körperlichen Training bei Patienten mit obstruktiven Atemwegserkrankungen. Pneumologie 2000; 54: 61 -67

21 American Thoracic Society. ATS Statement: Guidelines for the six-minute Walk Test. Am J Resp Crit Care Med 2002; 166: 111 - 117

22 Büsching G, Hilfiker R, Mangold F, Messmer G. Assessments in der Rehabilitation. Band 3: Kardiologie und Pneumologie. Bern: Huber, 2009

23 Gollner E, Kreuzriegler F, Kreuzriegler K. Rehabilitatives Ausdauertraining. München: Pflaum, 1991

24 Lagerström $C$, Nordgren $B$. On the reliability and usefulness of methods for grip strength measurement. Scand J Rehabil Med 1998; 30: 113 119

25 Oesch P, Hilfiker R, Keller S, Kool J. Assessments in der muskuloskeletalen Rehabilitation. Bern: Huber, 2007

26 Taube K. Sport- und Bewegungstherapie bei chronischen Lungenerkrankungen mit Berücksichtigung der COPD. In: Dalichau S, Scheele $\mathrm{K}$, Hrsg. Prävention und Rehabilitation durch Sport. Butzbach-Griedel: Afra, 2006: $14-30$

27 Bullinger M, Kirchberger I. SF-36. Fragebogen zum Gesundheitszustand. Göttingen: Hogrefe, 1998

28 Huber G. Sporttherapeutisches Assessment. In: Schüle K, Huber G, Hrsg. Grundlagen der Sporttherapie. 2. Aufl. München: Urban\&Fischer, 2004: 121 - 133

29 Enright PL, Sherrill DL. Reference equations for the six-minute walk in healthy adults. Am J Respir Crit Care Med 1998; 158: 1384-1387

30 Rost R, Hollmann W. Belastungsuntersuchungen in der Praxis. Stuttgart: Thieme, 1982

31 Mathiowetz V, Kashman N, Volland G et al. Grip and pinch strength: normative data for adults. Arch Phys Med Rehabil 1985; 66: 69-74

32 Ulmer WT, Nolte D, Lecheler J, Schäfer T, Hrsg. Die Lungenfunktion. Methodik und klinische Anwendung. 7. Aufl. Stuttgart: Thieme, 2003

33 Brehm W, Eberhardt J. Drop-out und Bindung im Fitness-Studio. Sportwissenschaft 1995; 25: 174-185

34 Fuchs R. Körperliche Bewegung und Psychologie. Göttingen: Hogrefe, 1997

35 Pahmeier I. Barrieren vor und Bindung an gesundheitssportliche Aktivität. In: Bös K, Brehm W, Hrsg. Gesundheitssport. Hamburg: Czwalina, 1998: $124-134$

36 Pahmeier I. Sportliche Aktivität als Bewältigungsaufgabe bei gesundheitlichen Beeinträchtigungen. Frankfurt a.M: Deutsch, 1994

37 Rampf J. Drop-out und Bindung im Fitness-Sport. Hamburg: Czwalina, 1999

38 Göhl O. Grundlagen der Trainingstherapie bei COPD. Atemw-Lungenkrkh 2005; 31: $501-509$

39 Brehm W, Pahmeier I, Tiemann M. Gesund und Fit: Gesundheitsprogramme für Erwachsene. Schorndorf: Hofmann, 2001

40 Köpke K-H. Aufwerten, ausbauen und systematisieren - Eine Analyse von Situation, Reformbedarf und innovativen Projekten zur Nachsorge in der Rehabilitation der Rentenversicherung. Rehabilitation 2005; 44: $344-352$

41 Bundesarbeitsgemeinschaft für Rehabilitation, Hrsg. Praxisleitfaden. Strategien zur Sicherung der Nachhaltigkeit von Leistungen zur medizinischen Rehabilitation. Frankfurt a.M: BAR, 2008 